EPJ Web of Conferences 59, 02012 (2013)

DOI: $10.1051 /$ epjconf/20135902012

(C) Owned by the authors, published by EDP Sciences, 2013

\title{
Ignition capsules with aerogel-supported liquid DT fuel for the National Ignition Facility
}

\author{
D.D.-M. Ho , J.D. Salmonson, D.S. Clark, J.D. Lindl, S.W. Haan, P. Amendt \\ and K.J. Wu
}

Lawrence Livermore National Laboratory, Livermore, CA 94550 USA

\begin{abstract}
For high repetition-rate fusion power plant applications, capsules with aerogel-supported liquid DT fuel can have much reduced fill time compared to $\beta$-layering a solid DT fuel layer. The melting point of liquid DT can be lowered once liquid DT is embedded in an aerogel matrix, and the DT vapor density is consequently closer to the desired density for optimal capsule design requirement. We present design for NIF-scale aerogel-filled capsules based on 1-D and 2-D simulations. An optimal configuration is obtained when the outer radius is increased until the clean fuel fraction is within $65-75 \%$ at peak velocity. A scan (in ablator and fuel thickness parameter space) is used to optimize the capsule configurations. The optimized aerogel-filled capsule has good low-mode robustness and acceptable high-mode mix.
\end{abstract}

\section{INTRODUCTION}

The achievement of ignition at the National Ignition Facility (NIF) is anticipated in the near term based on current experimental progress [1]. The next step is the demonstration of inertial fusion energy for power plant applications [2]. For high repetition-rate target injection in proposed fusion power plant applications, the solid-DT fuel, used in NIF capsules, will be replaced by liquid DT that is supported by a low-density $-20-30 \mathrm{mg} / \mathrm{cm}^{3}, \mathrm{C}_{10} \mathrm{H}_{12}$ dicyclopentadienyl (DCPD), nanoporous aerogel ("wettedaerogel"). Liquid DT provides a much reduced fill time compared to $\beta$-layering as well as lowering the tritium inventory. However, introducing aerogel has both positive and negative impacts on capsule performance. This paper shows that a systematic approach for optimizing the configuration and x-ray drive of wetted-aerogel capsules can lead to acceptable performance.

\section{ADVANTAGES AND DISADVANTAGES OF REPLACING SOLID DT WITH WETTED-AEROGEL}

Using aerogel to support liquid DT has both advantages and disadvantages.

\subsection{Advantages}

(1) Surface tension created by the porous structure in aerogel can lower the melting point as observed in liquid hydrogen [3]. If the aerogel can also lower the melting point of liquid DT, then the liquidDT vapor density can be reduced to below the solid-DT vapor density of $0.3 \mathrm{mg} / \mathrm{cm}^{3}$ used in NIF capsules. A lower DT vapor density, e.g., $0.15 \mathrm{mg} / \mathrm{cm}^{3}$, improves the robustness of ignition [4]. (Note that for solid DT, we cannot obtain vapor density as low as $0.15 \mathrm{mg} / \mathrm{cm}^{3}$ since this requires lowering the temperature of solid DT considerably below the triple point, which results in groove formation on the solid-DT surface [5]).

This is an Open Access article distributed under the terms of the Creative Commons Attribution License 2.0, which permits unrestricted use, distribution, and reproduction in any medium, provided the original work is properly cited. 


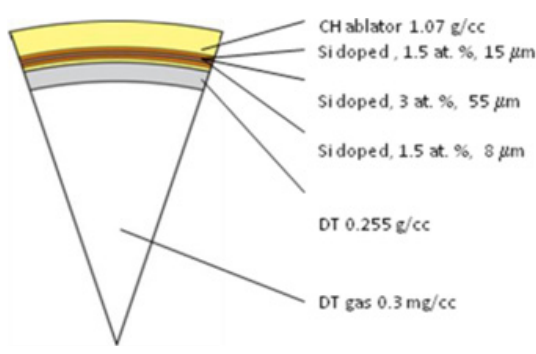

outer radius $=1.108 \mathrm{~mm}$ ablator thickness $=192 \mathrm{~m}$ fuel thickness $=72.2 \mu \mathrm{m}$ fuel mass $=0.17 \mathrm{mg}$

Figure 1. Configuration of the NIF scale pure-DT capsule with plastic ablator.

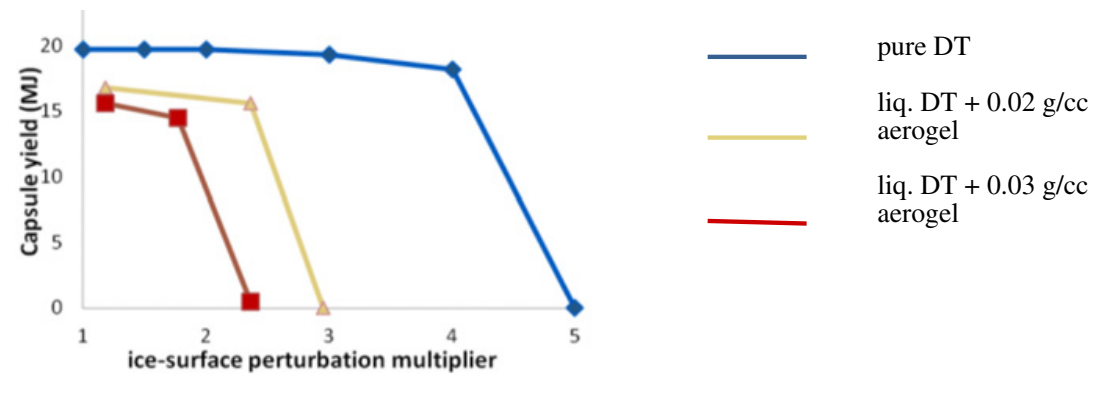

Figure 2. Low-mode robustness of NIF scale pure-DT vs wetted-aerogel capsules with same outer radius.

(2) Aerogel surface can possibly be smoother than solid DT surface because: (a) refinement in wet gel coating processes allows the fabrication of smoother DCPD aerogel surface, and (b) roughness may be smoothed out once aerogel is conformally filled with liquid DT.

\subsection{Disadvantages}

(1) For the same fuel mass, the payload mass for wetted-aerogel capsule is increased. An increased payload mass reduces the peak velocity and therefore the robustness.

(2) Since liquid DT has lower density $\left(0.225 \mathrm{~g} / \mathrm{cm}^{3}\right)$, the fuel layer becomes thicker for the same fuel mass if the outer radius of the fuel layer remains the same as that of the pure-DT capsule. This also reduces the peak velocity.

(3) Carbon atoms in aerogel absorb radiation and raise fuel entropy.

(4) Carbon in aerogel increases radiation loss during fuel assembly.

Optimizing the capsule performance, based on the understanding of these advantages and disadvantages, is described in the following section.

\section{A SYSTEMATIC APPROACH TO OPTIMIZE WETTED-AEROGEL CAPSULES}

We started with the NIF-scale, pure-DT capsule with plastic ablator (see Fig. 1). The NIF pure-DT capsule has an outer radius of $1.108 \mathrm{~mm}$ ("ref. radius"), absorbs $167 \mathrm{~kJ}$, and has a yield of $19.4 \mathrm{MJ}$.

This pure-DT capsule has good low-mode $(l \leq 30)$ behavior as shown in Fig. 2. It is also robust to high-mode $(l \leq 1000)$ mix at the ablator-fuel interface, e.g. the clean fuel fraction is $83 \%$ at peak velocity. Since the dominant perturbation at the ablator-fuel interface is around mode 60 and there is only a $1-2 \%$ difference in the clean fuel fraction between simulations with maximum mode of 200 and mode 1000, we will rely on simulations that include only low and intermediate modes, i.e., mode numbers up to 200 , to estimate the amount of mix at the ablator-fuel interface. 


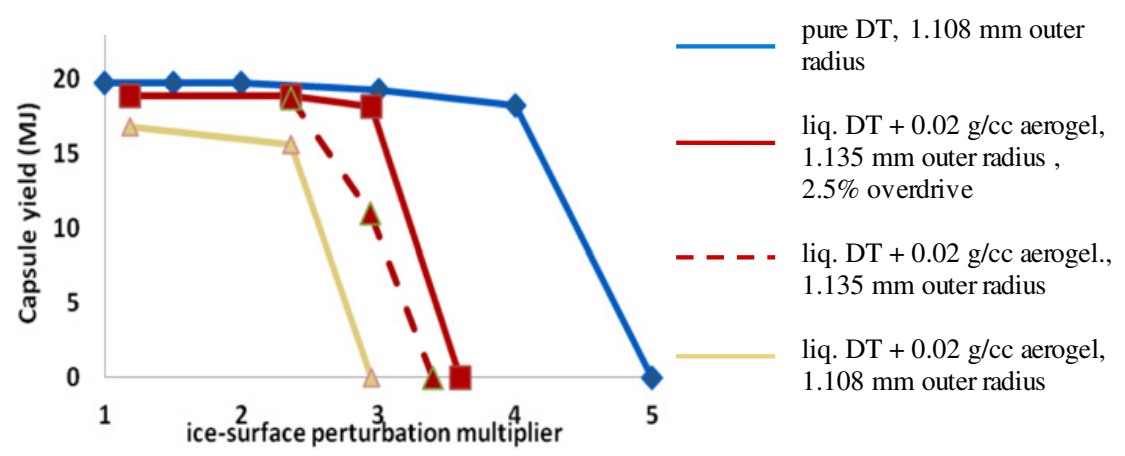

Figure 3. Low-mode robustness of NIF pure-DT vs wetted-aerogel capsules with different outer radii.

If we replace the pure-DT fuel in this capsule by a wetted-aerogel with an aerogel density of $20 \mathrm{mg} / \mathrm{cm}^{3}$, at the same payload mass (mass of liquid DT + foam mass) and same DT gas density, then the yield drops by more than $10 \%$. Even worse, the wetted-aerogel capsule has considerably less low-mode robustness than the pure-DT capsule as shown in Fig. 2 (based on 2-D low-mode hydro simulations with realistic surface roughness and driven by a radiation temperature source). This figure also indicates that the low-mode robustness deteriorates rapidly as aerogel density increases. For this reason, the focus of our study will be based on an aerogel with density at $20 \mathrm{mg} / \mathrm{cm}^{3}$ (the density of liquid DT + aerogel at $20 \mathrm{mg} / \mathrm{cm}^{3}$ is $0.2405 \mathrm{~g} / \mathrm{cm}^{3}$ ).

To recover from the lost/lower yield and the reduced implosion velocity, we increase the wettedaerogel capsule radius by $2.4 \%$ from the ref. radius to $1.135 \mathrm{~mm}$ while maintaining the same fuel mass of $0.17 \mathrm{mg}$. With the presence of the $20 \mathrm{mg} / \mathrm{cm}^{3}$ aerogel, the payload mass is increased to $0.184 \mathrm{mg}$. To ensure that there is enough ablator mass remaining and enough clean mass fraction at the time of peak velocity, the ablator and the heavily doped layer thicknesses are also increased to 195 and $60 \mathrm{~m}$, respectively. With increased ablator thickness, the initial aspect ratio of this larger capsule is about the same as that for the NIF pure-DT capsule. The 1-D yield of this larger capsule is only $3 \%$ less than that of the pure-DT capsule and the absorbed energy increases to $178 \mathrm{~kJ}$.

The low-mode robustness of this larger wetted-foam capsule is plotted in Fig. 3 against the robustness of the NIF pure-DT capsule and the wetted-aerogel capsule with the same outer radius as the pure-DT capsule. As shown in this figure, a larger radius results in increased robustness. The robustness can be further improved, if this capsule is given a $2.5 \%$ "overdrive", i.e., the radiation flux at peak temperature is increased by $2.5 \%$, as shown by the solid red line. However, a larger capsule and applying such an overdrive can result in increased high-mode mix. We therefore carried out intermediate-mode simulations to investigate the mix.

For the larger radius wetted-foam capsule without overdrive, the clean fuel fraction at peak velocity decreases from $83 \%$, for the NIF pure-DT capsule, to $80 \%$ which is within the Rev 5 specification that calls for a clean fuel fraction to be within $65-75 \%$ to maintain robustness. Therefore, the optimized configuration for a wetted-aerogel capsule is an increased capsule radius until the clean fuel fraction approaches $65-75 \%$. Simulations for capsules with overdrive will be presented shortly.

Exploring the potential of obtaining lower DT vapor density by lowering the liquid-DT temperature further improves the robustness as shown by the green curve in Fig. 4. Intermediate mode simulations show that reducing the DT gas density from 0.3 to $0.15 \mathrm{mg} / \mathrm{cm}^{3}$ reduces the clean fuel fraction at peak velocity from 80 to $77 \%$ and increases the convergence ratio from 39 to 45 . A $4 \%$ overdrive further reduces the clean fuel fraction to $72 \%$ which still satisfies the Rev5 mix requirement.

To further improve the capsule performance, we need to perform a "Herrmann-type" scan [6]. This scheme automatically optimizes the shock timing as the ablator and fuel thicknesses vary while the 

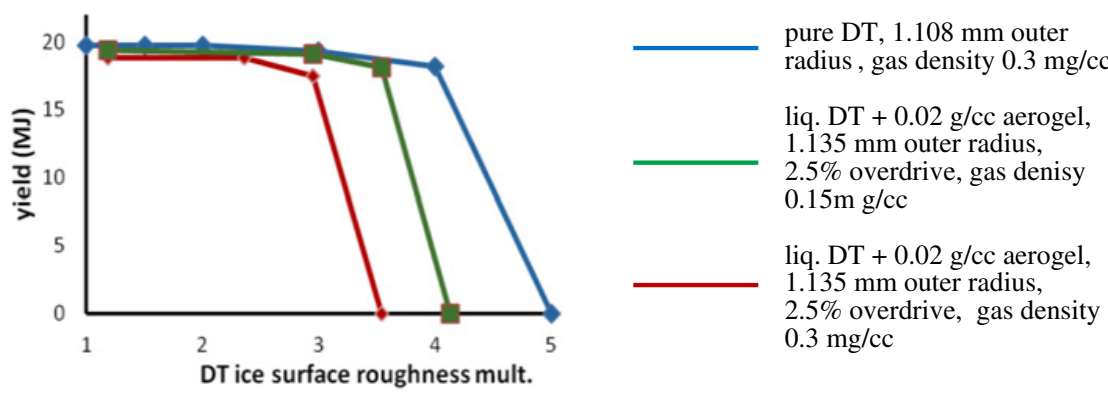

Figure 4. Low-mode robustness of pure-DT vs wetted-aerogel capsules with various DT vapor densities.

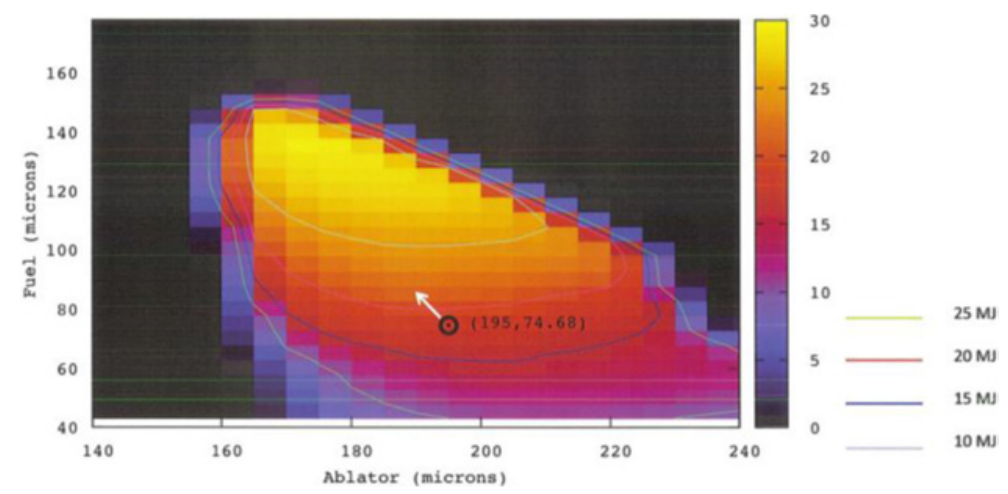

Figure 5. Yield contours from the Herrmann scan for wetted-aerogel capsules with aerogel density of $0.02 \mathrm{~g} / \mathrm{cc}$ and outer radius of $1.135 \mathrm{~mm}$.

capsule outer radius remains unchanged. The yield contours as a function of ablator and fuel thicknesses are shown in Fig. 5. Although this scan is based on 1-D calculations, it can provide guidance for 2-D mix by over plotting the contours of ablator mass remaining on Fig. 5 .

In this figure, the black circle is the location of our reference wetted-aerogel capsule described above and the white arrow is the direction of a capsule configuration change (ablator changes from 195 to $190 \mu \mathrm{m}$ and fuel layer changes from 74.7 to $83 \mu \mathrm{m}$ ) for improving the performance, as suggested by the scan. This configuration change improves the 1-D performance margin, entropy, and yield. However, the Atwood number and ablator mass remaining are slightly worse, which suggests an increase in mix that is confirmed by intermediate mode simulations. A detailed description of optimizing this capsule configuration using the Herrmann scan will be described in a forthcoming paper.

The capsule optimized by the scan, with a $4 \%$ overdrive, has a slightly higher yield of $20.9 \mathrm{MJ}$, while the low-mode robustness remains the same as shown by the green curve in Fig. 4. The clean fuel fraction drops from 72 to $67 \%$. The absorbed energy is $176 \mathrm{~kJ}$, which represents a $5.4 \%$ increase from the original NIF pure-DT capsule. This increase in absorbed energy for recovering the capsule performance from the presence of aerogel can potentially be recovered from high-efficiency advanced hohlraum designs [7].

\section{CONCLUSION}

We have described a systematic method for optimizing the robustness and yield for capsules with wetted aerogels. By using a lower DT vapor density, slightly larger capsule radius and higher peak 
drive, simulations of wetted-aerogel capsules show good low-mode robustness and acceptable clean fuel fraction with essentially no deterioration in yield compared to NIF pure-DT capsules. Further improvements in robustness are possible if the aerogel surface roughness can be further reduced.

This work performed under the auspices of the U.S. Department of Energy by Lawrence Livermore National Laboratory under Contract DE-AC52-07NA27344.

\section{References}

[1] J. D. Lindl, $7^{\text {th }}$ IFSA Conf., Bordeaux (2011)

[2] M. Dunne, Sci. and Tech. Review, Lawrence Livermore Nat'l Lab., July/Aug. (2011)

[3] G. Beaudoin, Journal of Low Temp. Phys. 105 (1996)

[4] S. W. Haan et al., Phys. Plasmas 18 (2011)

[5] S. O. Kucheyev and A. V. Hamza, J. of Applied Phys. 108 (2010)

[6] M. C. Herrmann, personal communication (2005)

[7] P. Amendt, M. Dunne, D. D. Ho, and J. D. Lindl, Fus. Sci. Tech. 60 (2011) 